\title{
Traum und Trauma
}

\section{Erhard Taverna}

Die Ausstellung in Bonn über «Napoleon und Europa Traum und Trauma» wird, ohne Verlängerung, am 25. April 2011 zu Ende gehen. Wer einen Besuch verpasst hat, bekommt von März bis Juni 2012 in Paris noch einmal eine Chance zu einer überaus lohnenden Geschichtslektion. Die Folgen, die sich aus der 16-jährigen Herrschaft Napoleons ergaben, sind Gegenstand der zwölf thematischen Stationen in der rheinischen Bundeskunsthalle.

Verherrlichung oder Verdammnis, das Urteil über die Epoche des Korsen fiel in den 200 Jahren seit dem Russlandfeldzug auch ausserhalb Frankreichs nie neutral aus. Dazu gibt es viele Beispiele: Während England 2005 ein gigantisches Spektakel zu Ehren der siegreichen Seeschlacht von Trafalgar inszenierte, ignorierte das offizielle Frankreich im gleichen Jahr die Schlacht von Austerlitz. Erst nach den Studentenunruhen von 1968 zelebrierte de Gaulle einen hagiographischen Rundgang zum wundersamen Schicksal des heldenhaften Bonaparte. Seither gab es zahlreiche Ausstellungen von Italien bis Moskau, doch stets aus vorwiegend regionaler oder nationaler Perspektive.

Heute gelten neue Positionen der Geschichtsbetrachtung. In Bonn geht es erstmals um die grossen europäischen Zusammenhänge, mehr um das Verstehen als das Gedenken. Den Machern ist es gelungen, die komplexe Geschichte auf eine gut verständliche Art $\mathrm{zu}$ verdichten. Dabei gilt die Aufmerksamkeit neben dem Biografischen und den Koalitionskriegen auch ungewohnteren Aspekten wie der neuen Administration, dem Strassenbau, der Post und Telegrafie, dem ausgedehnten Kunstraub und der gezielten Bildpropaganda aller kriegsführenden Parteien. Europa

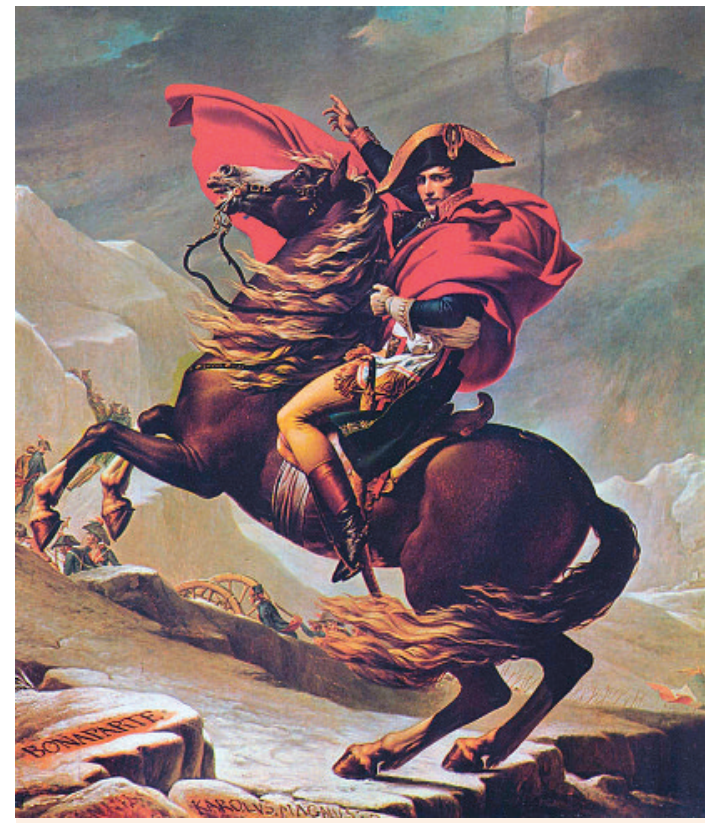

Napoleon als Held: Auf dem Gemälde von Jacques-Louis David aus dem Jahr 1800 überschreitet er die Alpen am Grossen Sankt Bernhard.

erhalten. Die Feldzüge verursachten etwa 5 Millionen Tote und doppelt so viel Verletzte. 2002 stiessen Bagger in einem Neubauviertel von Vilnius auf 18 Skelette, in denen man zuerst Opfer der Nazis oder des KGB vermutete. Litauens führender Anthropologe und Anatom identifizierte sie als Elitesoldaten der Grande Armée, der mit 600000 Mann und 160000 Pferden grössten aller bisherigen Armeen. Die Küras-

\section{Erstmals geht es um die grossen europäischen Zusammenhänge, mehr um das Verstehen als das Gedenken.}

wurde modernisiert, vieles von den Siegern übernommen, etwa der "Code Napoléon», den das Regime in der Zeit von 1807 bis 1814 in allen dominierten Staaten einführte. In mehrfach revidierter Fassung gilt der Code in Frankreich bis heute. Dichter wie Heine und Stendhal erfreuten sich an dessen Sprache, Juristen loben den selbsternannten Kaiser als genialen Rechtstheoretiker. Der Verfasser selbst führte seinen bleibenden Ruhm auf sein Gesetzeswerk und nicht auf die 40 gewonnenen Schlachten zurück.

Ruhm und Ehre sind das eine, das andere sind die siere lagen auf den Massengräbern von weiteren 35000 Toten, darunter wohl auch Gefallene der 9000 mitmarschierten Eidgenossen, von denen 700 wieder nach Hause kamen. Die medizinische Fakultät untersuchte die Gebeine. In 435 Fällen gelang eine Altersbestimmung, die Hälfte der Gefallenen war 20-25 Jahre alt.

Was Stachanow im Russland Stalins, war der Kürassier Chipault in Frankreich nach dem siegreichen Polenfeldzug 1807. Nach vorbildlichen Einsätzen wurde er mit 52 chirurgisch beglaubigten Verletzungen durch Säbel- und Lanzenhiebe zum hoch- 


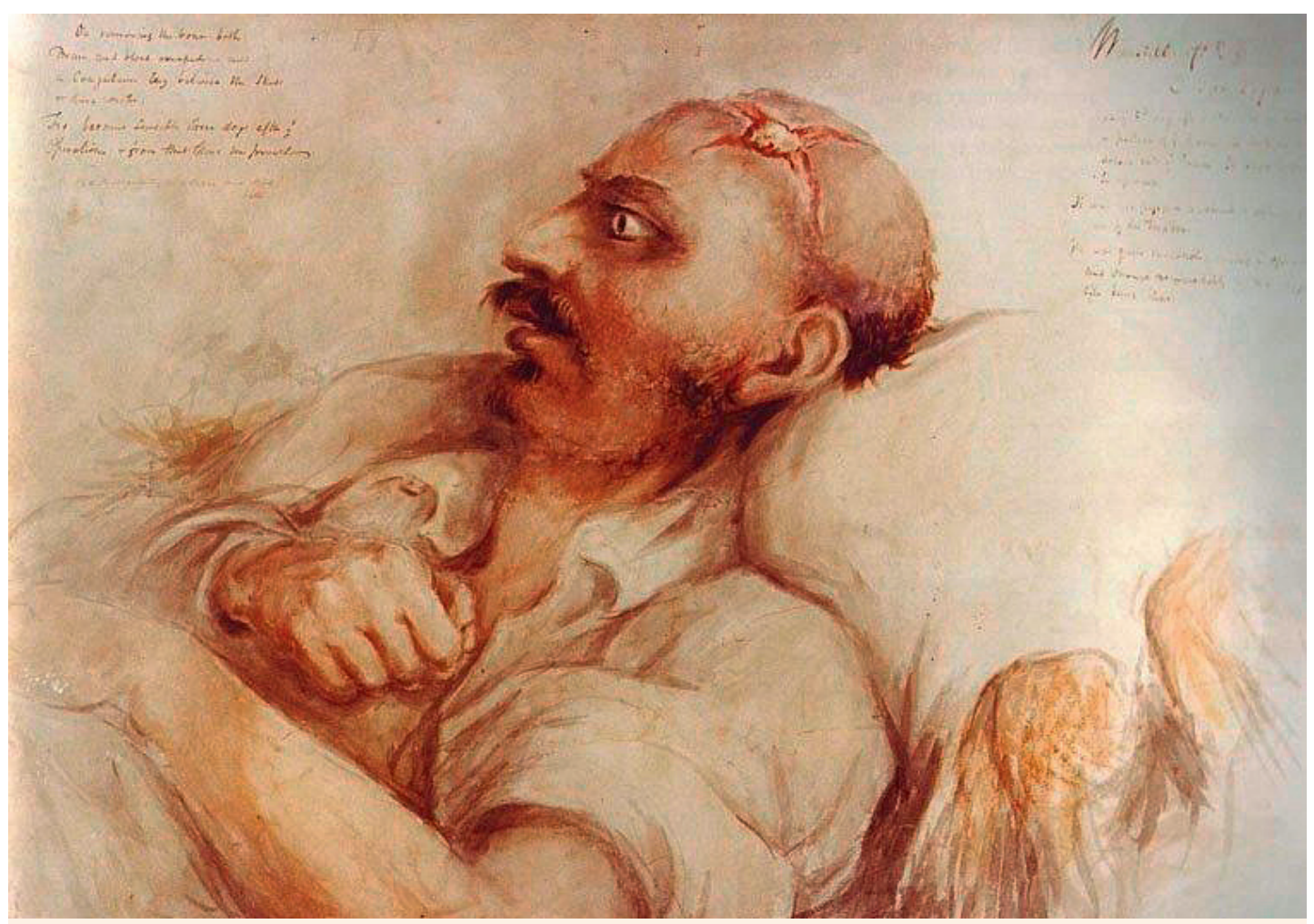

Der Anatom und Physiologe Charles Bell (1774-1842), der auch in Waterloo Kriegsversehrte versorgte, hielt in seinem Skizzenheft die Schrecken des Krieges fest.

dekorierten Vorzeigehelden, an allen Aufmärschen herumgereicht, in Heeresberichten vorgeführt und literarisch verwertet. Auf die Situation der damaligen Kriegsverletzten verweisen zahlreiche Abbildungen und Exponate. Starrkrampf, Gasbrand, Pest, Typhus und Cholera brachten ebenso viele ins Grab wie Schusswunden und Stichverletzungen. Der in Waterloo tätige Anatom und Physiologe Charles Bell (1774-1842) besass neben seinen chirurgischen Instrumenten ein Skizzenheft, in dem er zahlreiche Verwundungen dokumentierte. Vier seiner aufgehängten Aquarelle zeigen Kopfwunden, Schockzustände und amputierte Gliedmassen im drastischen Gegensatz zur pathetischen Salonmalerei jener Jahre. Sein Name hat als «Bell'sches Phänomen» in den Neurologiebüchern überlebt. Zwei ballistische Gelatineblöcke demonstrieren die Wirkung von Rundkugelgeschossen aus Vorderladern. Die tiefen, grossflächig zerfetzten Wundhöhlen boten ideale Bedingungen für Infektionen. Ohne Antibiotika und ohne Anästhesie verhalf nur die schnelle Amputation zu einer Überlebenschance.

Der grosse Star jener Zeit war Baron Jean Dominique Larrey (1766-1842), Stabsarzt der Rheinarmee und Chefarzt der Grande Armée, Erfinder der mobilen Feldambulanzen. Ein Ölbild zeigt den Dekorierten in Uniform, wie er einem Sitzenden den linken Oberarm durchtrennt. Die Ambulanzwagen von Larrey mit ihrem Team waren ein grosser Fortschritt. Sie beschleunigten die medizinische Hilfe, die er auch gegnerischen Verletzten und Offizieren wie Soldaten gleichermassen leistete. Seine berittenen «ambulances volantes» wurden sofort kopiert und waren später Vorbild für die amerikanischen «Mobile Army Surgical Hospitals (MASH)». Neben den geöffneten Koffern und Futteralen mit Trepanations-Instrumenten und Chirurgenbesteck liegen Hand- und Beinprothesen und als Besonderheit auch eine Zahnprothese aus sogenannten «Waterloo-Zähnen», die besonders krass die Realität des Krieges zeigt. Leichenfledderer brachen den Toten und Sterbenden die Zähne aus dem Mund, die als Zahnersatz mit vernieteten Metallstiften auf Elfenbein montiert wurden. Larrey hat alle Feldzüge mitgemacht, in der Schlacht von Borodino soll er persönlich 200 Amputationen aus dem Gelenk durchgeführt haben. Seine Doktorarbeit «Mémoire sur les amputations» aus dem Jahr 1803 wurde als Standardwerk mehrfach aufgelegt. Als selbstloser und humaner Mensch war er bei der Truppe hoch angesehen. Er nahm vieles vorweg, was später Henri Dunant verwirklichte.

Die medizinischen Fakten sind nur ein Kapitel im zentralen Raum zum Thema «Der Traum vom Weltreich». Die Fülle der erwähnten Informationen soll einen Begriff von der enormen Vielfalt dieser Ausstellung vermitteln. Das hervorragend gestaltete, umfangreiche Buch zur Ausstellung [1] bietet mit Essays und einem Anhang eine unentbehrliche Verdauungshilfe.
Traum und Trauma. München, Berlin, London, New York: Prestel; 2010. 